\title{
The Galactic Cepheid period-luminosity relation revisited using bona fide cluster Cepheids
}

\author{
Richard I. Anderson, Nami Mowlavi, and Laurent Eyer \\ Observatoire de Genève, Université de Genève, 51 Ch. des Maillettes, CH-1290 Versoix, \\ Switzerland \\ email: richard.anderson@unige.ch
}

\begin{abstract}
Classical Cepheids in Galactic open clusters (cluster Cepheids: CCs) have been studied extensively for multiple decades, thanks to their importance as calibrators of the Galactic Cepheid period-luminosity relation (PLR). Here we revisit the calibration of the Galactic PLR using a new sample of CCs, since even recent calibrations show significant discrepancies. The $\mathrm{CC}$ sample employed for the calibration is based on the preliminary results of a self-consistent, eight-dimensional all-sky census. This census is based mostly on literature data, supplemented with high-precision radial-velocity observations from both hemispheres. New CCs are identified from our census and the degree of confidence in membership is quantified for known candidates. Using only bona fide CCs, we obtain $M_{V}=(-3.08 \pm 0.50) \log P+(-0.94 \pm 0.42) \mathrm{mag}$, which is in perfect agreement with the results by Sandage, Tammann, and Reindl, albeit with larger error bars and an rms of $0.21 \mathrm{mag}$. The key to obtaining a meaningful calibration is to employ accurate cluster distance moduli and space reddening values. A homogeneous study of all bona fide host clusters would be desirable to increase precision and confidence in the calibration.
\end{abstract}

Keywords. Cepheids, open clusters and associations: general, stars: distances, distance scale

\section{Introduction}

This year marks the centennial anniversary of the discovery of period-luminosity (density) relationships by Leavitt \& Pickering (1912), who identified this important proportionality in a sample of 25 periodic variable stars identified among 1777 stars in the Small Magellanic Cloud. A century later, Cepheids and other pulsating stars are corner stones of the distance scale and commonly used to determine distances of up to tens of Mpc, and this Symposium had many excellent examples that this can be done at impressive precision. Nevertheless, there exist significant differences between PLRs found in the (recent) literature, possibly indicating less consensus on the accuracy of these measurements than sometimes acknowledged. We illustrate this fact in Fig. 1, which shows PLRs based on different methods, including absolute and relative trigonometric parallaxes, BaadeBecker-Wesselink-type methods, as well as the calibration by Sandage et al. (2004) which is partially based on Cepheids in Galactic open clusters. In light of such discrepancies, we deem that a self-consistent analysis of membership for CCs is warranted, and here we present a calibration obtained from the preliminary results of our mostly literaturebased, eight-dimensional, self-consistent all-sky census of classical Cepheids in Galactic open clusters. 


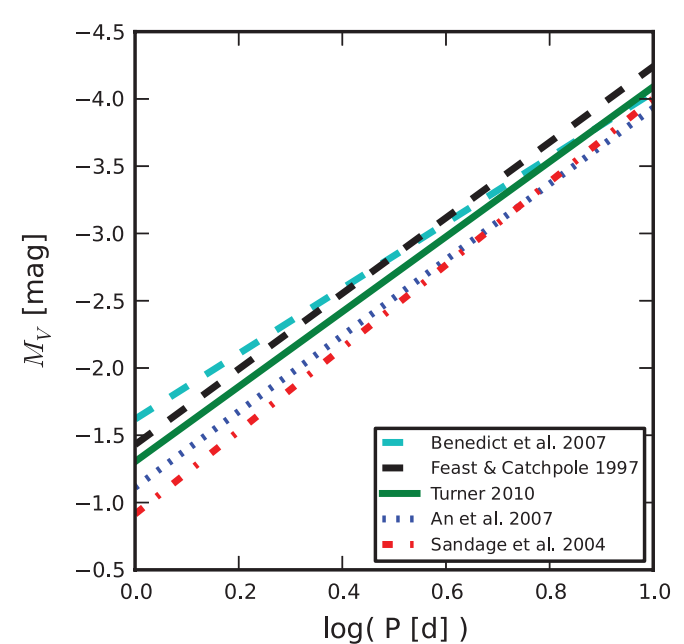

Figure 1. Literature PLRs, see legend

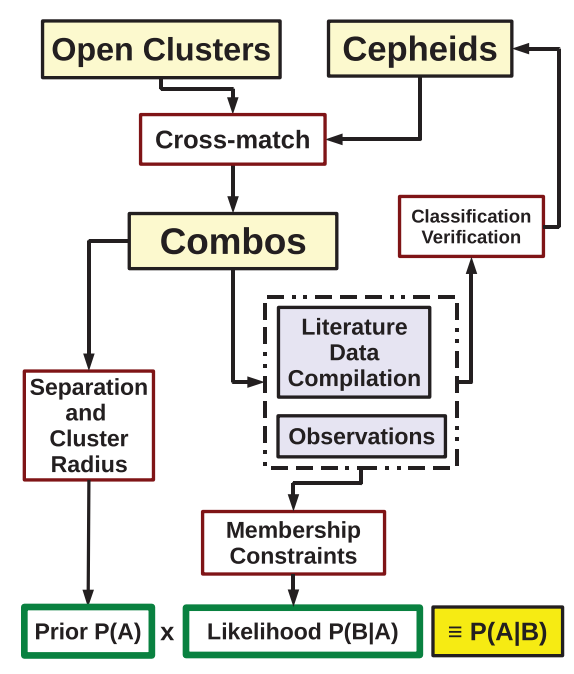

Figure 2. Membership analysis overview

\section{The Cluster Cepheid Sample}

Fig. 2 schematically shows how the all-sky membership census for CCs is set up. Starting from a cross-match of known open clusters and Cepheids, a prior (from the position on the sky and the cluster radius) and a likelihood (using all other available data) are used to calculate membership probabilities. The data used to calculate the likelihoods is mostly from published literature, and supplemented by observations taken at the Swiss Euler telescope at ESO La Silla, Chile, and at the Mercator telescope at the Roque de los Muchachos Observatory on La Palma, Spain.

The membership census is eight-dimensional, employing the three spatial dimensions, proper motion, radial velocity, iron abundance as proxy for metallicity, and the age as constraints. A total of 2570 cluster-Cepheid combinations were investigated with the aim of identifying new CCs and quantifying the degree of confidence one can have in the membership of CC candidates previously discussed in the literature. We identify 13 bona fide members of open clusters, three of which are pulsating in their first overtones. Unfortunately, our analysis is somewhat limited by the open cluster data available, so that another 12 CCs known in Turner (2010) could not be revisited. We ignore these cases here, and stick to the highest-confidence CCs for the calibration.

Another important part of the process is classification verification through photometry and spectroscopy, since we systematically include Cepheid candidates from automated surveys such as ASAS, NSVS, and ROTSE that are not yet confirmed as Cepheids.

The sample of CCs used for the PLR calibration are the 10 bona fide CCs that pulsate in the fundamental mode. These constitute the cluster Cepheids in which we can have the highest confidence of membership, based on our self-consistent study. For details on the analysis and the sample used (including the observing program, the new CC calibrators, etc.), we refer to our publication in preparation (R. I. Anderson et al., in prep.).

\section{The PLR calibration}

Since cluster membership has been thoroughly established for the Cepheids used in this calibration, the independently (from the Cepheids) determined cluster distances can be used to obtain a Galactic calibration of the PLR, in particular of its zero point. As 


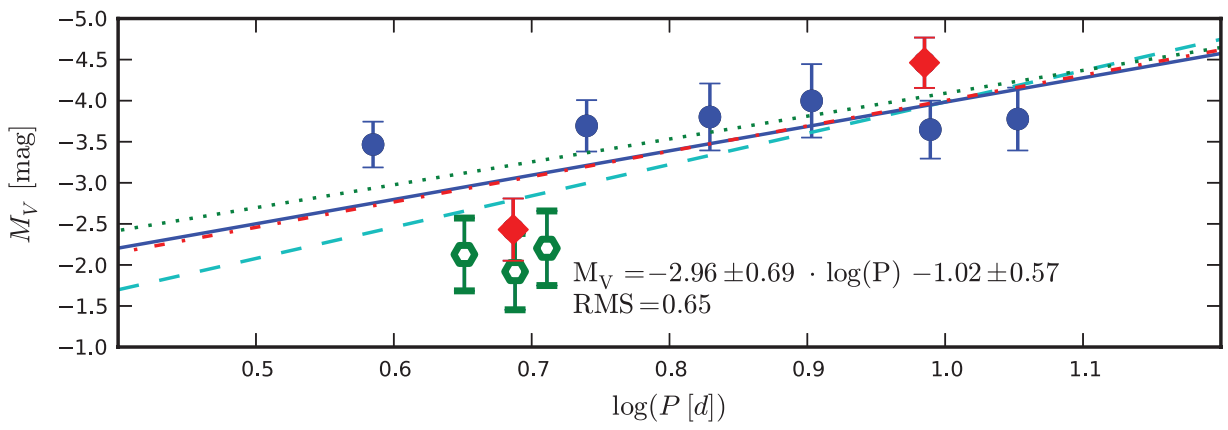

Figure 3. Solid blue line: weighted least-squares fit to the 'standard' data set (cluster data based on fewer stars, no line-of-sight dependence of reddening); red dash-dotted line: non-weighted fit; dotted green line: PLR from Benedict et al. (2007); dashed cyan line: PLR from Sandage et al. (2004).

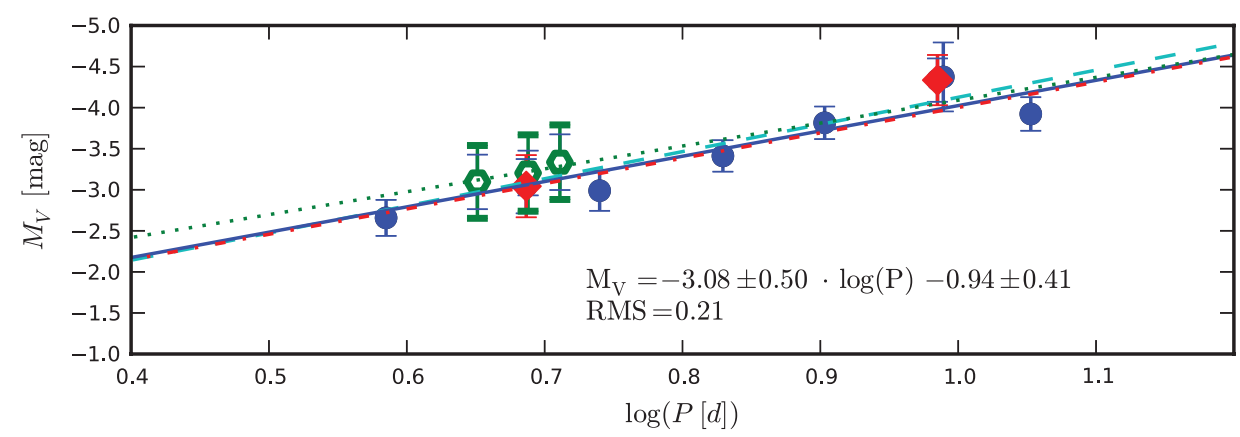

Figure 4. Solid blue line: weighted least-squares fit to the 'optimal' data set (clusters studied in detail individually, line-of-sight-dependent reddening law); red dash-dotted line: non-weighted fit; dotted green and dashed cyan lines: as in Fig. 3.

is clear from Fig. 1, this remains an important task, and is complicated by the small number of cluster Cepheids, as well as by the difficulties in determining accurate and precise cluster distances and space reddening values.

Obviously, the Cepheids' estimated absolute magnitudes, $M_{V}$, depend critically on the clusters' distance moduli and reddening values adopted. Fig. 3 shows $M_{V}$ calculated using distance moduli from catalogs containing many hundreds of clusters. Such semiautomated and often model-dependent (isochrone-based) studies do not take into account line-of-sight variations in the reddening law and typically use the canonical $R \equiv 3.1$. In addition, they are often limited in photometric depth and therefore determine distances (and ages) using relatively few stars. A great benefit of such studies, however, is selfconsistency and homogeneity. We refer to these $M_{V}$ magnitudes as the 'standard' set.

Fig. 4, in contrast, shows the absolute magnitudes estimated using line-of-sight-dependent reddening laws and distance moduli based on deeper photometric studies found in the literature. Unfortunately, no homogeneous detailed study could be found that provides these data for the entire sample of host clusters. However, most host clusters have been studied previously and we can therefore manually select references we deem optimal. This manual compilation of references aims to maximize homogeneity and detail (number of stars studied, treatment of reddening). No criterion to favor any existing PLR calibration was imposed. We therefore refer to this set of $M_{V}$ values as the 'optimal' set, since the greatest accuracy can be assumed for these values. 
The errors in $M_{V}$ are estimated by taking into account uncertainties in reddening and an ad hoc estimate of the distance modulus. Green open hexagons represent Cepheids in NGC 7790 studied by Gupta et al. (2000), and have slightly larger error bars to avoid excessive weight on data points that depend on a single cluster's values for reddening and distance modulus. Solid blue circles represent other CCs previously discussed in the literature. The two new cluster Cepheids (to be identified in R. I. Anderson et al., in prep.) employed in this calibration will benefit greatly from a more detailed study of their host clusters. For the time being, we employ $R=3.1$ and the distance modulus given in the original literature. They are marked as solid red diamonds in Figs 3 and 4.

We perform weighted and non-weighted least-squares fits to both data sets (standard and optimal). The results between weighted and non-weighted fits are very similar for both data sets. As can be expected, we obtain large scatter ( $\mathrm{rms}=0.65 \mathrm{mag}$ ) for the standard data set. The optimal set exhibits less scatter ( $\mathrm{rms}=0.21 \mathrm{mag}$ ) around the relation $M_{V}=(-3.08 \pm 0.50) \log P+(-0.94 \pm 0.42)$ mag, with considerable uncertainty in both the zero point and the slope. We note that our result is in near exact agreement with the PLR in Sandage et al. (2004), although nearly all of the PLRs shown in Fig. 1 agree with our results to within their uncertainties. However, the discrepancy between the PLRs becomes dramatic at longer periods $(P>10$ days $)$ and can significantly impact the distance scale, since longer-period Cepheids are more luminous and are, therefore, seen to greater distances. The zero point of the PLR hence remains of key importance and a calibration like ours has the potential to contribute significantly. However, additional refinement is required, and can be achieved through detailed studies of Cepheid host clusters, as well as through continued efforts to discover CCs.

\section{Conclusion}

This work clearly shows the need for continued studies of the Cepheid PLR within our own Galaxy. Our approach aims to maximize the number of CCs that can be employed for the PLR calibration through an unprecedented eight-dimensional all-sky census that self-consistently evaluates the degree of confidence one can have in cluster membership, thereby ensuring that only the best CCs are used in the calibration. Unfortunately, the precision of our results remains limited by the small number of bona fide CCs and the relatively uncertain open cluster data.

A good way to improve on our results will be to carry out a homogeneous reddening and distance modulus determination for all clusters in the sample. In less than a year from now, Gaia will be launched. Providing homogeneous positions, astrometry, photometry and for some stars even spectroscopy, Gaia will provide unprecedented astrometric precision that will revolutionize membership studies such as ours. Based on Gaia data, it will thus be possible to significantly improve our membership analysis and enable an all-sky, and much more homogeneous study of the kind presented here.

\section{References}

An, D., Terndrup, D. M., \& Pinsonneault, M. H. 2007, ApJ, 671, 1640

Benedict, G. F., McArthur, B. E., Feast, M. W., et al. 2007, AJ, 133, 1810

Feast, M. W., \& Catchpole, R. M. 1997, MNRAS, 286, L1

Gupta, A. C., Subramaniam, A., Sagar, R., \& Griffiths, W. K. 2000, A\&AS, 145, 365

Leavitt, H. S. \& Pickering, E. C. 1912, Harvard Coll. Obs. Circ., 173, 1

Sandage, A., Tammann, G. A., \& Reindl, B. 2004, A\&SA, 424, 43

Turner, D. G., Mandushev, G. I., \& Welch, G. A. 1997, AJ, 113, 2104

Turner, D. G. 2010, ApESSS, 326, 219 